\title{
Editorial
}

\section{Virtual Airway Assessment-So Far and Yet So Close!}

\author{
Karen Ruby Lionel ${ }^{1}$ \\ ${ }^{1}$ Department of Neuroanaesthesia, Christian Medical College, \\ Vellore, Tamil Nadu, India
}

J Neurosci Rural Pract 2021;12:611-612.

Coronavirus disease 2019 (COVID-19) has brought into focus the occupational hazards that healthcare workers face. Since the risk of acquiring aerosol-transmitted diseases is directly proportional to the proximity to patients and the length of time spent within a potentially infectious area, anesthetists who deal with airway assessment and management are particularly at risk of infection. ${ }^{1}$ The recognition of these risks has led to several adaptations and innovations to decrease exposure to aerosols. These include intubation using an aerosol box, creating a negative pressure theater environment, adopting rapid sequence intubation protocols using video-laryngoscopes, and modifying the basic and advanced cardiac life support protocols. Telecommunication tools have been widely adopted for patient interaction in telemedicine consultations and conferencing between medical professionals. COVID-19 has necessitated restrictions on movement for people residing in poorly connected rural areas of the developing world. However, the rapid expansion of Internet access and penetration of smartphones offer an unique opportunity to bridge the health access gaps.

Hrishi, in this edition of the journal, presents an interesting, low-cost application of telemedicine in preanesthetic assessment relevant to resource-poor settings. COVID-19 transmission occurs efficiently from asymptomatic and presymptomatic individuals and poses a particular challenge during the preanesthetic assessment. ${ }^{2}$ An accurate airway assessment, an essential component of the preanesthetic check, requires the patient to remove their mask, exposing the examining anesthetist to the risk of close contact with the patient's respiratory tract and secretions. This study demonstrates that smartphone-based airway assessment can decrease the anesthetist's exposure to coronavirus without compromising patient care.

The role of telemedicine in the preoperative assessment with a focus on comorbidities and their optimization as well as airway assessment has been described earlier and is in practice in many centers where distance is the issue. ${ }^{3-5} \mathrm{~A}$ similar but simplified principle, the use of smartphones, using the direct-to-consumer visit model instead of the brick-and-mortar teleconsultation site, has been used by Hrishi in this study to evaluate the efficacy of virtual versus direct airway assessment in the preoperative period in patients presenting for neurosurgery. 2,6

Additionally, as succinctly described by Bridges et al, telemedicine has applications beyond the immediate threat of COVID-19 and these need to be explored carefully. ${ }^{6}$ Where access to perioperative physicians is constrained, telemedicine offers an alternative to provide prehabilitation virtually. ${ }^{7}$ Use of these innovative measures may be exploited by anesthetists to promote remote preoperative and intraoperative guidance to optimize comorbidities and to manage as appropriate. ${ }^{8}$ Likewise, the transition of care from inhospital to a postoperative follow-up at home, be it surgical or an anesthetic follow-up regarding the titration of medications or optimizing postoperative pain relief, can also be performed virtually, preventing avoidable readmissions. ${ }^{9}$ This timely publication in the Journal of Neurosciences in Rural Practice (JNRP) emphasizes smartphone-based secure assessment modalities, which can be extrapolated further to the entire perioperative period.

However, looking at this in a different light, with all its myriad benefits, remote communication has some significant shortcomings compared with traditional means of communication. Patients, especially those awaiting surgery, experience anxiety and benefit from the empathetic interaction that a preoperative consultation provides. As technological solutions grow, we must remain cognizant that these tools, while useful, do not replace human touch and direct interaction. While communicating virtually, the physician must strive to maintain a good rapport and communication with the patient to recompense the effects of an understanding handshake, a reassuring smile, a hand on the shoulder which we conventionally and often subconsciously use during a physical encounter with the patient. Furthermore, we
Address for correspondence Karen Ruby Lionel, MD, DM, Department of

Neuroanaesthesia, Christian

Medical Collage, Ida Scudder

Road, Vellore, Tamil Nadu

632004, India, India

(e-mail: kanjacob@gmail.com).
DOI https://doi.org/ 10.1055/s-0041-1736152. ISSN 0976-3147. (c) 2021. Association for Helping Neurosurgical Sick People. All rights reserved.

This is an open access article published by Thieme under the terms of the Creative Commons Attribution-NonDerivative-NonCommercial-License, permitting copying and reproduction so long as the original work is given appropriate credit. Contents may not be used for commercial purposes, or adapted, remixed, transformed or built upon. (https://creativecommons.org/ licenses/by-nc-nd/4.0/)

Thieme Medical and Scientific Publishers Pvt. Ltd., A-12, 2nd Floor, Sector 2, Noida-201301 UP, India 
must also be cognizant of confidentiality issues, secure storage, transmission of medical images, and national laws that need to be adhered to while developing solutions. Innovation is the need of the hour, and this timely seasonable publication in the JNRP shows that technology offers solutions for these unprecedented times. Nonetheless, we must remember that care is more than a clinical examination and the balance must always be held.

\section{Conflict of Interest}

None declared.

\section{References}

1 Irons JF, Pavey W, Bennetts JS, Granger E, Tutungi E, Almeida A. COVID-19 safety: aerosol-generating procedures and cardiothoracic surgery and anaesthesia - Australian and New Zealand consensus statement. Med J Aust 2021;214(01):40-44

2 Hrishi AP, Prathapadas P, Praveen R, Vimala S, Sethuraman M. A comparative study to evaluate the efficacy of virtual versus direct airway assessment in the preoperative period in patients presenting for neurosurgery. A quest for safer preoperative practice in neuro anesthesia in the backdrop of the COVID19 pandemic!. J Neurosci Rural Pract 2021;12(4):718-725

3 Schoen DC, Prater K. Role of telehealth in pre-anesthetic evaluations. AANA J 2019;87(01):43-49

4 Galvez JA, Rehman MA. Telemedicine in anesthesia: an update. Curr Opin Anaesthesiol 2011;24(04):459-462

5 Chatrath V, Attri JP, Chatrath R. Telemedicine and anaesthesia. Indian J Anaesth 2010;54(03):199-204

6 Bridges KH, McSwain JR, Wilson PR. To infinity and beyond: the past, present, and future of tele-anesthesia. Anesth Analg 2020; 130(02):276-284

7 Barberan-Garcia A, Ubré M, Roca J, et al. Personalised prehabilitation in high-risk patients undergoing elective major abdominal surgery: a randomized blinded controlled trial. Ann Surg 2018; 267(01):50-56

8 Kamdar N, Jalilian L. Telemedicine: a digital interface for perioperative anesthetic care. Anesth Analg 2020;130(02): 272-275

9 Williams AM, Bhatti UF, Alam HB, Nikolian VC. The role of telemedicine in postoperative care. mHealth 2018;4:11 